University of South Carolina

Scholar Commons

9-15-2005

Persistent and Radiation-Induced Currents in Distorted Quantum Rings

Yuriy V. Pershin Dr

University of South Carolina - Columbia, pershin@physics.sc.edu

Carlo Piermarocchi

Follow this and additional works at: https://scholarcommons.sc.edu/phys_facpub

Part of the Physics Commons

Publication Info

Published in Physical Review B, ed. Gene D. Sprouse, Volume 72, Issue 12, 2005, pages

125348-1-125348-7.

Pershin, Y. V., \& Piermarocchi, C. (2005). Persistent and radiation-induced currents in distorted quantum rings. Physical Review B, 72(12), 125348-1 - 125348-7. DOI: 10.1103/PhysRevB.72.125348

(C) Physical Review B, 2005, American Physical Society

This Article is brought to you by the Physics and Astronomy, Department of at Scholar Commons. It has been accepted for inclusion in Faculty Publications by an authorized administrator of Scholar Commons. For more information, please contact digres@mailbox.sc.edu. 


\title{
Persistent and radiation-induced currents in distorted quantum rings
}

\author{
Yuriy V. Pershin and Carlo Piermarocchi \\ Department of Physics and Astronomy, Michigan State University, East Lansing, Michigan 48824-2320, USA
}

(Received 6 June 2005; revised manuscript received 20 July 2005; published 30 September 2005)

\begin{abstract}
Persistent and radiation-induced currents in distorted narrow quantum rings are theoretically investigated. We show that ring distorsions can be described using a geometrical potential term. We analyze the effect of this term on the current induced by a magnetic flux (persistent current) and by a polarized coherent electromagnetic field (radiation-induced current). The strongest effects in persistent currents are observed for distorted rings with a small number of electrons. The distortion smoothes the current oscillations as a function of the magnetic flux and changes the temperature dependence of the current amplitude. For radiation-induced currents, the distortion induces an ac component in the current and affects its dependence on the radiation frequency and intensity.
\end{abstract}

DOI: 10.1103/PhysRevB.72.125348

PACS number(s): 73.23.Ra

\section{INTRODUCTION}

It is well known that a static magnetic flux through a mesoscopic ring induces a dissipationless nondecaying (persistent) current at low temperature. During the last 20 years this persistent current has been heavily investigated from both the theoretical and experimental sides. ${ }^{1-22}$ In particular, theoretical investigations were focused on the effects of electron-electron interaction, disorder, spin-orbit, ${ }^{2}$ polarized nuclear spins, ${ }^{3,4}$ and shape. ${ }^{19,20}$ More recently, the effect of electromagnetic radiation on mesoscopic rings has been investigated. It has been pointed out that the persistent current can be strongly affected by radiation ${ }^{21}$ and also that radiation can induce a current at zero magnetic flux. ${ }^{22,23}$ In order to break the clockwise-anticlockwise symmetry and obtain a current at zero flux, a radiation with some degree of circular polarization is needed. This can be achieved by a superposition of pairs of time-asymmetric, linearly crosspolarized picosecond pulses, ${ }^{23}$ or more simply by using circularly polarized radiation. ${ }^{22}$ All these effects were mainly studied in systems with perfect ring geometry, i.e., rings where the confinement potential does not depend on the azimuthal coordinate. The only exceptions, to our knowledge, are Refs. 19 and 20, where the persistent current in an elliptical quantum ring $^{19}$ and the persistent current in a quantum ring on a surface of constant negative curvature ${ }^{20}$ were considered.

In this paper we investigate persistent and radiationinduced currents in distorted quantum rings. We consider a narrow distorted ring of uniform cross section lying on a plane (Fig. 1). The ring can be composed by several segments of different curvature or can have any smooth curvature profile. We show that the curvature of the ring enters into the Schrödinger equation via a geometrical potential term of the form $V_{\text {geom }}=-\hbar^{2} /\left(8 m^{*} R^{2}\right)$, where $R$ is the radius of curvature. Our model differs from the one used in Ref. 19, where a quantum ring of a nonuniform cross section was considered. By choosing a uniform cross section the effect of the distorsion can be described in a simpler and more transparent way. Our paper is organized as follows. In Sec. II we derive the Schrödinger equation for one electron in a distorted ring in the presence of a magnetic flux. We show that, as in the ideal case, the wave function in the distorted ring is a periodic function of the magnetic flux $\Phi$ with period $\Phi_{0}$. Next, in Sec. III, we consider a model where a distorted quantum ring consists of four constant-curvature segments. We find the energy spectrum of such a ring and we demonstrate that the geometrical potential $V_{\text {geom }}$ in this case opens gaps in the electron energy spectrum. Moreover, we show that the geometrical potential can lead to bound states. The oscillation of the persistent current, and the frequency and intensity dependence of the radiation-induced currents in the distorted ring are studied in Sec. IV. The results of our investigations are summarized in Sec. V.

\section{EFFECTIVE HAMILTONIAN}

We consider one electron with effective mass $m^{*}$ confined by a potential $V_{\gamma}\left(\gamma\right.$ denotes the characteristic width of $\left.V_{\gamma}\right)$ to

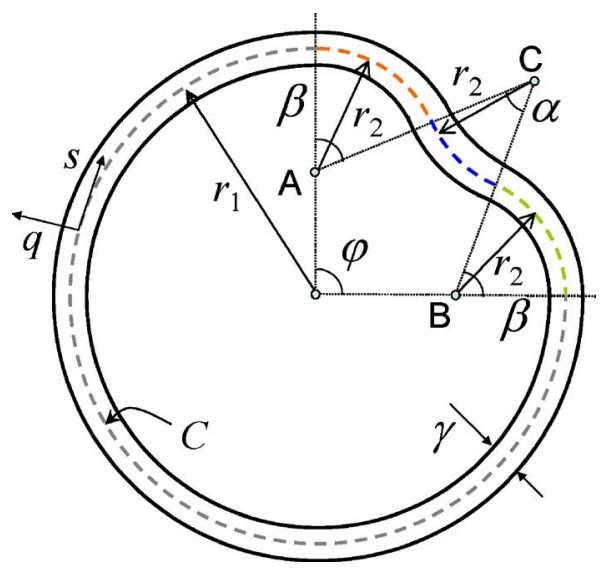

FIG. 1. (Color online) Distorted quantum ring consisting of a long segment of radius $r_{1}$ and three short segments of radius $r_{2}$. For a given angle $\varphi$, the point $C$ is obtained as the intersection point of two circumferences of the radius $2 r_{2}$ with the centers at $A$ and $B$. The shortest $r_{2}$ corresponds to the case when $A, B$, and $C$ are on the straight line. Therefore, it is easy to find that the minimum value of $r_{2}$ is given by $r_{2}^{\min }=\sin (\varphi / 2) r_{1} /[2+\sin (\varphi / 2)]$. For $\varphi=\pi / 4, r_{2}^{\min }$ $\simeq 0.16 r_{1}$. 
a closed curve $C$ on a plane. A uniform magnetic field $H$ perpendicular to the plane is applied. The Schrödinger equation has the form

$$
\frac{1}{2 m^{*}}\left(\hat{\mathbf{p}}-\frac{e}{c} \mathbf{A}\right)^{2} \psi+V_{\gamma} \psi=E \psi
$$

where $\hat{\mathbf{p}}$ is the electron momentum operator and $\mathbf{A}(\mathbf{r})$ $=\frac{1}{2}[\mathbf{H}, \mathbf{r}]$ is the vector potential. Using the property $\operatorname{div}(\mathbf{A})$ $=0$ of this gauge, Eq. (1) can be rewritten as

$$
\frac{1}{2 m^{*}}\left(-\hbar^{2} \Delta-2 \frac{e}{c} \mathbf{A} \hat{\mathbf{p}}+\frac{e^{2}}{c^{2}} \mathbf{A}^{2}\right) \psi+V_{\gamma} \psi=E \psi .
$$

Our goal is to obtain an effective one-dimensional Schrödinger equation in the limit of a strong transverse confinement, i.e., in the limit $\gamma \rightarrow 0$. We follow the approach proposed in Ref. 24 and subsequently used in Ref. 25.

Let us introduce the orthonormal coordinate system $(s, q)$, where $s$ is the arc length parameter and $q$ is the coordinate along the normal $\mathbf{n}(s)$. The curve $C$ is then described by a vector $\mathbf{r}(s)$ as a function of the arc length $s$. In a vicinity of $C$ the position is therefore is described by

$$
\mathbf{R}(s, q)=\mathbf{r}(s)+q \mathbf{n}(s) .
$$

For the sake of simplicity ${ }^{25}$ we assume that $V_{\gamma}$ depends only on the $q$ coordinate describing the displacement from the reference curve $C$ only.

The Laplacian $\Delta$ in the curvilinear coordinates $s$ and $q$ is given by

$$
\Delta_{s, q}=\frac{1}{h} \frac{\partial}{\partial s} \frac{1}{h} \frac{\partial}{\partial s}+\frac{1}{h} \frac{\partial}{\partial q} h \frac{\partial}{\partial q},
$$

with

$$
h=1-k(s) q,
$$

where $k(s)=R^{-1}(s)$ is the curvature. Using the transformation to the new wave function $\chi(s, q)$ via $\psi(s, q)=\chi(s, q) / \sqrt{h}$ [note, that $\chi(s, q)$ is properly normalized], we can rewrite Eq. (2) as

$$
\begin{aligned}
\frac{1}{2 m^{*}} & \left\{-\hbar^{2}\left(\frac{\partial}{\partial s} \frac{1}{h^{2}} \frac{\partial}{\partial s}-\frac{h_{s s}}{2 h^{3}}+\frac{5 h_{s}^{2}}{4 h^{4}}+\frac{\partial^{2}}{\partial q^{2}}+\frac{k^{2}}{4 h^{2}}\right)\right. \\
& +2 i \frac{e \hbar}{c}\left[A_{s}(s, q)\left(\frac{\partial}{\partial s}+\frac{k_{s} q}{2 h}\right)\right. \\
& \left.\left.+A_{q}(s, q)\left(\frac{\partial}{\partial q}+\frac{k}{2 h}\right)\right]+\frac{e^{2}}{c^{2}} \mathbf{A}^{2}\right\} \chi+V_{\gamma} \chi=E \chi,
\end{aligned}
$$

where $h_{s}=\partial h / \partial s, \quad h_{s s}=\partial^{2} h / \partial s^{2}, \quad k_{s}=\partial k / \partial s, A_{q}(s, q)$ and $A_{s}(s, q)$ are the components of $\mathbf{A}$ along the $q$ and $s$ directions. Next, we make the substitution

$$
\chi=\exp \left[i \frac{e}{\hbar c} \int_{0}^{q} A_{q}\left(s, q^{\prime}\right) d q^{\prime}\right] \tilde{\chi},
$$

and expand $h, A_{q}(s, q), A_{s}(s, q)$ in series in $q$ keeping only the zero-order terms in $q$, as in Refs. 24 and 25 . The Schrödinger equation (6) can then be easily separated by setting $\widetilde{\chi}(s, q)$ $=\nu(q) \phi(s)$. The usual procedure yields

$$
\begin{gathered}
-\frac{\hbar^{2}}{2 m^{*}} \frac{\partial^{2} \nu}{\partial q^{2}}+V_{\gamma} \nu=E_{t} \nu \\
{\left[-\frac{\hbar^{2}}{2 m^{*}} \frac{\partial^{2}}{\partial s^{2}}+i \frac{e \hbar}{m^{*} c} A_{s}(s, 0) \frac{\partial}{\partial s}-\frac{\hbar^{2} k^{2}}{8 m^{*}}-\frac{i e \hbar}{2 m^{*} c}\right.} \\
\left.\times \frac{\partial A_{q}(s, 0)}{\partial q}+i \frac{e \hbar}{m^{*} c} A_{q}(s, 0) \frac{k}{2}+\frac{e^{2}}{2 m^{*} c^{2}} A_{s}^{2}(s, 0)\right] \phi=E_{l} \phi .
\end{gathered}
$$

In order to further simplify Eq. (8), we perform the transformation

$$
\phi(s)=\exp \left[i \frac{e}{\hbar c} \int_{0}^{s} A_{s}\left(s^{\prime}, 0\right) d s^{\prime}\right] \widetilde{\phi}(s),
$$

which gives

$$
\begin{aligned}
& {\left[-\frac{\hbar^{2}}{2 m^{*}} \frac{\partial^{2}}{\partial s^{2}}-\frac{\hbar^{2} k^{2}}{8 m^{*}}-\frac{i e \hbar}{2 m^{*} c} \frac{\partial A_{s}(s, 0)}{\partial s}-\left.\frac{i e \hbar}{2 m^{*} c} \frac{\partial A_{q}(s, q)}{\partial q}\right|_{q=0}\right.} \\
& \left.\quad+i \frac{e \hbar}{m^{*} c} A_{q}(s, 0) \frac{k}{2}\right] \widetilde{\phi}(s)=E_{t} \widetilde{\phi}(s) .
\end{aligned}
$$

Notice that in the curvilinear coordinates $(s, q)$ the divergence of $\mathbf{A}$ is given by

$$
\operatorname{div} \mathbf{A}=\frac{1}{h} \frac{\partial}{\partial_{s}}\left(\frac{1}{h} A_{s}\right)+\frac{1}{h} \frac{\partial}{\partial q}\left(h A_{q}\right) \approx \frac{\partial}{\partial_{s}} A_{s}+\frac{\partial}{\partial q} A_{q}-k A_{q}=0 .
$$

Consequently, Eq. (9) reduces to

$$
-\frac{\hbar^{2}}{2 m^{*}} \frac{\partial^{2} \widetilde{\phi}}{\partial s^{2}}-\frac{\hbar^{2} k^{2}(s)}{8 m^{*}} \widetilde{\phi}=E_{l} \widetilde{\phi}
$$

Therefore, we have derived two decoupled equations: one describing the transverse confinement of electrons in the ring [Eq. (7)], and the second describing the longitudinal motion of the electron in the ring [Eq. (11)]. The vector potential A does not explicitly appear in these two equations. However it will appear in the solution of Eq. (11) because of the boundary conditions on $\widetilde{\phi}$ specified below. The spectrum of Eq. (7) depends on the particular shape of the confinement potential $V_{\gamma}$. In this paper we assume that the electrons occupy only the lowest subband of the transversal confinement. Therefore, the position of this energy subband is not important. The curvature of $C$ enters into Eq. (11) through the geometrical potential term $-\hbar^{2} k(s)^{2} / 8 m^{*}$.

The boundary conditions for $\widetilde{\phi}$ are obtained from the requirements of continuity of the wave function $\phi(s)$ and its derivative, i.e., $\phi(0)=\phi(L), \partial \phi(0) / \partial s=\partial \phi(L) / \partial s$ ( $L$ is the ring circumference). Using Stokes' theorem we finally obtain

$$
\widetilde{\phi}(0)=e^{i 2 \pi\left(\Phi / \Phi_{0}\right)} \widetilde{\phi}(L), \quad \frac{\partial \widetilde{\phi}(0)}{\partial s}=\frac{\partial \widetilde{\phi}(L)}{\partial s} .
$$

Here $\Phi$ is the magnetic flux through the area confined by $C$ and $\Phi_{0}$ is the magnetic flux quantum. Eqs. (12) imply that all equilibrium physical properties of a narrow closed loop are 

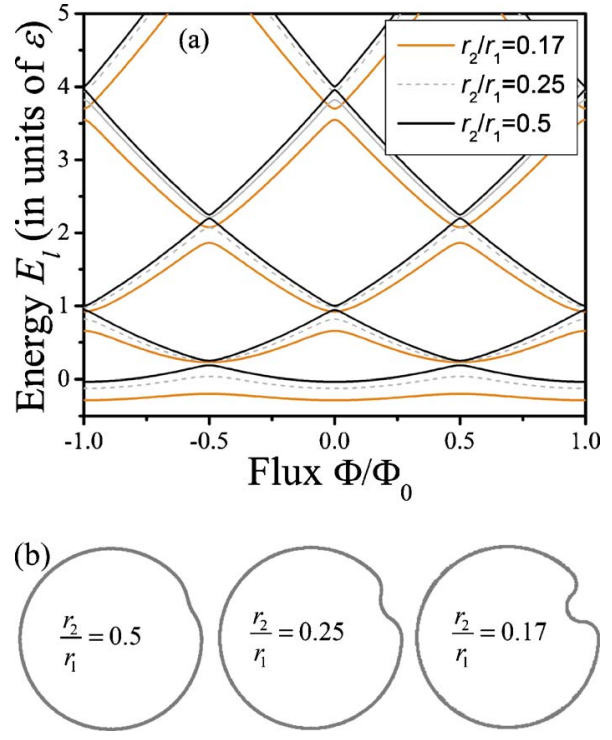

FIG. 2. (Color online) (a) Electron energy levels [in units of $\varepsilon$ $\left.=\hbar^{2} /\left(2 m^{*} r_{1}^{2}\right)\right]$ as a function of flux in quantum rings with different degrees of distortion at $\varphi=\pi / 4$. (b) Geometrical shape of the distorted quantum rings whose electron energy levels are shown in (a).

periodic in $\Phi$ with period $\Phi_{0}$, as in the case of a perfect ring. ${ }^{8}$

\section{ELECTRON ENERGY SPECTRUM}

In this section we consider the electron spectrum in a ring with a dent-type distortion as shown in Fig. 1. Geometrically, the curve $C$ of such a ring consists of four smoothly connected circular segments. The radius of the long segment is $r_{1}$, the three short segments have the same radius $r_{2}$ (we assume here that $r_{2}<r_{1}$ ). The angles $\alpha$ and $\beta$ are related to $\varphi$ (for the definition of these angles see Fig. 1) as $\alpha$ $=2 \operatorname{Arcsin}\left[\sin (\varphi / 2)\left(r_{1}-r_{2}\right) /\left(2 r_{2}\right)\right], \quad \beta=\varphi / 2+\alpha / 2$. The advantage of a distortion with constant curvature segments is a constant geometrical potential in each segment. The picture is even more simple since the geometrical potential does not depend on the direction of bending, i.e., $V_{\text {geom }}$ is the same for the three short segments in Fig. 1. Therefore, we write Eq. (11) for the long segment and three short segments as

$$
\begin{gathered}
-\frac{\hbar^{2}}{2 m^{*}} \frac{\partial^{2} \phi_{1}}{\partial s^{2}}-U_{0} \phi_{1}=E_{l} \phi_{1} \quad \text { for } 0<s<l, \\
-\frac{\hbar^{2}}{2 m^{*}} \frac{\partial^{2} \phi_{2}}{\partial s^{2}}=E_{l} \phi_{2} \quad \text { for } l<s<L,
\end{gathered}
$$

where $l=(2 \beta+\alpha) r_{2}$ is the total length of the short segments, $L=(2 \pi-\varphi) r_{1}+l, U_{0}=\left(\hbar^{2} / 8 m^{*}\right)\left(1 / r_{2}^{2}-1 / r_{1}^{2}\right)$. The general solution of Eqs. (13) and (14) reads

$$
\begin{aligned}
& \phi_{1}=b_{1} e^{i k_{1} s}+b_{2} e^{-i k_{1} s}, \\
& \phi_{2}=c_{1} e^{i k_{2} s}+c_{2} e^{-i k_{2} s},
\end{aligned}
$$

where $k_{1}=\sqrt{\left(2 m^{*} / \hbar\right)\left(E_{l}+U_{0}\right)}$ and $k_{2}=\sqrt{\left(2 m^{*} / \hbar\right) E_{l}}$. The wave functions $\phi_{1(2)}$ are connected at $s=l$ via $\phi_{1}(l)=\phi_{2}(l)$, $\partial \phi_{1}(l) / \partial s=\partial \phi_{2}(l) / \partial s$ and at $l=0, L$ via Eqs. (12). From these boundary conditions we obtain a transcendental equation defining the energy spectrum for unbound states $E_{l}>0$

$$
\begin{gathered}
2 \cos \left(2 \pi \frac{\Phi}{\Phi_{0}}\right)+\left[\frac{k_{1}}{k_{2}}+\frac{k_{2}}{k_{1}}\right] \sin \left(k_{1} l\right) \sin \left[k_{2}(L-l)\right] \\
-2 \cos \left(k_{1} l\right) \cos \left[k_{2}(L-l)\right]=0
\end{gathered}
$$

and for bound states $-U_{0}<E_{l}<0$

$$
\begin{aligned}
2 \cos \left(2 \pi \frac{\Phi}{\Phi_{0}}\right)+\left[\frac{k_{1}}{\tilde{k}_{2}}-\frac{\tilde{k}_{2}}{k_{1}}\right] \sin \left(k_{1} l\right) \sinh \left[\widetilde{k}_{2}(L-l)\right] \\
-2 \cos \left(k_{1} l\right) \cosh \left[\tilde{k}_{2}(L-l)\right]=0 .
\end{aligned}
$$

Here, $\tilde{k}_{2}=\sqrt{\left(2 m^{*} / \hbar\right)\left(-E_{l}\right)}$.

The calculated energy levels for weakly and strongly distorted rings as a function of the flux are given in Fig. 2. It is well known that in perfect rings the energy levels are intersecting parabolas. In distorted rings, gaps are opened at the points of intersection of the parabolas. This effect is qualitatively similar to the effect of disorder. ${ }^{8}$ We emphasize that for a fixed radius of distortion, the gap decreases for larger values of the intersection point energy. At a fixed point of intersection, the gap increases by decreasing the radius of distortion (i.e., the gap is larger in more distorted rings). Notice finally that due to the distortion the effective circumference of the ring increases. This produces negative shifts of the energy levels which are larger at higher energy. Notice in the spectrum the presence of bound states with $E_{l}<0$. Similar bound states were already discussed in elliptical quantum rings. ${ }^{19}$ It is interesting that the transition from unbound to bound states in the ring is smooth: the shallow bound states are still sensitive to the magnetic flux (Fig. 2, $r_{2} / r_{1}=0.25$ ). In contrast, deep bound states have a weak sensitivity to the magnetic flux (Fig. 2, $r_{2} / r_{1}=0.17$ ). Correspondingly, there is a finite contribution to the persistent current from the shallow bound states, while the contribution from the deep bound states is small.

\section{EFFECT OF THE DISTORTION ON THE CURRENT}

\section{A. Persistent currents induced by a magnetic flux}

We consider a system with a fixed number of spinless electrons $N$ and calculate the current using the standard approach. ${ }^{26,27}$ At nonzero temperature $T$, the current in the ring is given by

$$
I=-\frac{\partial F}{\partial \Phi}
$$

Here, the free energy $F=-k_{B} T \ln Z$, where $k_{B}$ is the Boltzman constant, $T$ is the temperature, and $Z=\Sigma_{n} \exp \left[-\epsilon_{n} /\left(k_{B} T\right)\right]$ is the partition function defined through the energies of manyparticle states $\epsilon_{n}$.

In Fig. 3, we show the effect of distortion on the persistent current in quantum rings with three electrons. These results were obtained numerically using Eq. (19) with the energy spectrum determined from Eqs. (17) and (18). At zero tem- 


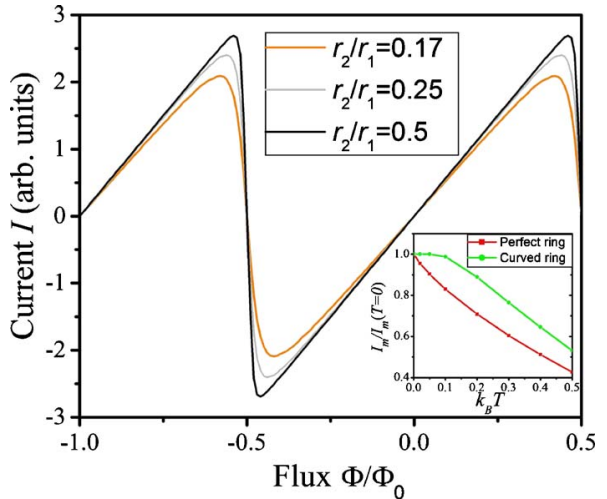

FIG. 3. (Color online) Persistent current in the ring at $T=0, \varphi$ $=\pi / 4, N=3$. Inset: temperature dependence of the persistent current amplitude in the perfect and distorted $\left(\varphi=\pi / 4, r_{2} / r_{1}=0.17\right)$ rings. $k_{B} T$ is in units of $\varepsilon$.

perature the persistent current oscillations in a perfect ring have a sawtooth form. The distortion of the ring produces a smoothing of the oscillations due to the opening of energy gaps at the intersection points. Figure 3 shows that the smoothing increases for larger distortions. Notice that the persistent current as a function of the magnetic field flux in the distorted ring at $T=0$ looks similar to the persistent current in a perfect ring at $T>0$.

The distortion of the ring changes the temperature dependence of the current amplitude. The temperature dependence of the persistent current and its amplitude in a perfect ring with a fixed number of electrons at low temperatures was derived using a two-level approach, which takes into account contributions from the ground and first excited levels to the partition function. It was found that at low temperatures the persistent current can be written as

$$
I=\frac{2 N \varepsilon}{\Phi_{0}}\left[\frac{\sinh \gamma}{1+\cosh \gamma}-2\left(\frac{\Phi}{\Phi_{0}}-\frac{\delta}{2}\right)\right]
$$

where $\gamma=2 N \varepsilon\left(\Phi / \Phi_{0}-\delta / 2\right) /\left(k_{B} T\right), \varepsilon=\hbar^{2} /\left(2 m^{*} r^{2}\right), \delta=0$ if $N$ is even and $\delta=1$ if $N$ is odd, and the overall factor 2 takes into account the spin degeneracy. The temperature dependence of the amplitude of the persistent current oscillations is given by

$$
I_{\max }=\frac{2 N \varepsilon}{\Phi_{0}}\left(\sqrt{1-2 \frac{k_{B} T}{N \varepsilon}}-\frac{k_{B} T}{N \varepsilon} \operatorname{arccosh}\left[\frac{N \varepsilon}{k_{B} T}-1\right]\right) .
$$

The comparison of the temperature dependence of the persistent current amplitude in the perfect and distorted rings is shown in the inset of Fig. 3. While the persistent current amplitude in the perfect ring starts to decrease at $T=0$, the temperature dependence in the distorted ring shows an activation energy behavior in the vicinity of $T=0$ due to the gaps in the energy spectrum.

In quantum rings with many electrons, the main contribution to the persistent current is due to the electrons near the Fermi level. Since the energy gap disappears for large values of the intersection point energy, the persistent current at
$T=0$ will not be smoothed. Consequently, the persistent current in a distorted ring with a large number of electrons will be as in a perfect ring with a radius $r^{*}=L /(2 \pi)$ in a weaker (for the case of a dent) magnetic field $B^{*}=B S / S^{*}$, where $S$ is the area of the distorted ring and $S^{*}=2 \pi r^{* 2}$.

\section{B. Current induced by circularly polarized radiation}

In a recent paper, ${ }^{22}$ a mechanism for current generation in quantum rings was proposed. It was suggested that in the presence of a circularly polarized continuous-wave (cw) radiation, the light-dressed ground state of the ring is characterized by a nonzero current. The purpose of this section is to study the influence of the ring distortion on this radiationinduced current.

Let us consider an electron confined in a distorted ring in the presence of circularly polarized $\mathrm{cw}$ radiation. The single electron Hamiltonian in the dipole approximation reads

$$
H=H_{0}+V(t)=-\frac{\hbar^{2}}{2 m^{*}} \frac{\partial^{2}}{\partial s^{2}}+U_{g e o m}(s)+e \mathbf{E}(t) \mathbf{r}(s),
$$

where $\mathbf{E}(t)=E_{0} \cos (\omega t) \hat{x} \pm E_{0} \sin (\omega t) \hat{y}$ is the circularly polarized electric field, $E_{0}$ is its amplitude, and \pm corresponds to $\sigma_{ \pm}$radiation. The distorted quantum ring is considered again as made of four constant curvature segments, which allows us to use the energy spectrum and wave functions of $H_{0}$ obtained in Sec. III at $\Phi=0$. Assuming that the radiation frequency is close to the transition between the ground and two first excited levels, we restrict our attention only to these three levels, with energies given by $E_{0}, E_{1}$ and $E_{2}$.

The external radiation causes transitions between these levels. The electron dynamics in the ring can be conveniently described using the a density matrix approach similar to the one used in Ref. 28 for quantum dots. The evolution of density matrix $\rho$ is given by

$$
i \hbar \dot{\rho}=[H, \rho]-\Gamma\{\rho\},
$$

where $\Gamma\{\rho\}$ represents relaxation terms. In the rotating wave approximation the corresponding equations for the density matrix elements are

$$
\dot{\rho}_{00}=v_{01-} \tilde{\rho}_{10}+v_{02-} \tilde{\rho}_{20}-\widetilde{\rho}_{01} v_{10+}-\tilde{\rho}_{02} v_{20+}+\kappa_{20} \rho_{22}+\kappa_{10} \rho_{11},
$$

$$
\begin{aligned}
& \dot{\rho}_{11}=v_{10+} \tilde{\rho}_{01}-\tilde{\rho}_{10} v_{01-}-\kappa_{10} \rho_{11}+\kappa_{21} \rho_{22}, \\
& \dot{\rho}_{22}=v_{20+} \tilde{\rho}_{02}-\tilde{\rho}_{20} v_{02-}-\kappa_{20} \rho_{22}-\kappa_{21} \rho_{22},
\end{aligned}
$$

$$
\dot{\tilde{\rho}}_{01}=\frac{E_{0}-E_{1}+\hbar \omega}{i \hbar} \widetilde{\rho}_{01}+v_{01-} \rho_{11}+v_{02-} \rho_{21}-\rho_{00} v_{01-}-\gamma_{01} \widetilde{\rho}_{01},
$$

$$
\dot{\tilde{\rho}}_{02}=\frac{E_{0}-E_{2}+\hbar \omega}{i \hbar} \widetilde{\rho}_{02}+v_{01-} \rho_{12}+v_{02-} \rho_{22}-\rho_{00} v_{02-}-\gamma_{02} \widetilde{\rho}_{02},
$$

$$
\dot{\rho}_{12}=\frac{E_{1}-E_{2}}{i \hbar} \rho_{12}+v_{10+} \widetilde{\rho}_{02}-\widetilde{\rho}_{10} v_{02-}-\gamma_{12} \rho_{12},
$$


$\dot{\tilde{\rho}}_{10}=\frac{E_{1}-E_{0}-\hbar \omega}{i \hbar} \widetilde{\rho}_{10}+v_{10+} \rho_{00}-\rho_{11} v_{10+}-\rho_{12} v_{20+}-\gamma_{10} \widetilde{\rho}_{10}$,

$\dot{\tilde{\rho}}_{20}=\frac{E_{2}-E_{0}-\hbar \omega}{i \hbar} \widetilde{\rho}_{20}+v_{20+} \rho_{00}-\rho_{21} v_{10+}-\rho_{22} v_{20+}-\gamma_{20} \widetilde{\rho}_{20}$,

$$
\dot{\rho}_{21}=\frac{E_{2}-E_{1}}{i \hbar} \rho_{21}+v_{20+} \tilde{\rho}_{01}-\widetilde{\rho}_{20} v_{01-}-\gamma_{21} \rho_{21} \text {. }
$$

Here, the transformations $\rho_{01}=e^{i \omega t} \widetilde{\rho}_{01}, \quad \rho_{02}=e^{i \omega t} \widetilde{\rho}_{02}, \quad \rho_{10}$ $=e^{-i \omega t} \widetilde{\rho}_{10}$, and $\rho_{20}=e^{-i \omega t} \widetilde{\rho}_{20}$ were used, $\kappa_{i j}$ is the relaxation rate of diagonal density matrix elements, and $\gamma_{i, j}$ is the dephasing rate of the off-diagonal coherences $\rho_{i j} . v_{i j \pm}$ $=\langle i|V(t)| j\rangle e^{ \pm i \omega t} /(i \hbar)$, where $\overline{(\ldots)}$ denotes an averaging over a period of $V(t)$. For example, in the case of $\sigma_{+}$radiation we obtain

$$
\begin{aligned}
& v_{01-}=\frac{1}{i \hbar} \frac{e E_{0}}{2}\left\langle 0\left|x+\frac{y}{i}\right| 1\right\rangle, \\
& v_{10+}=\frac{1}{i \hbar} \frac{e E_{0}}{2}\left\langle 1\left|x-\frac{y}{i}\right| 0\right\rangle .
\end{aligned}
$$

The persistent current is calculated using $I=\operatorname{Tr}[\rho \hat{j}]$, where $\hat{j}$ is the standard quantum mechanical current operator, and $\rho$ is the steady-state solution of Eqs. (24)-(32). We find that in the distorted rings the current operator matrix has a form

$$
\hat{j}=\left(\begin{array}{ccc}
0 & 0 & j_{02} \\
0 & 0 & j_{12} \\
j_{20} & j_{12} & 0
\end{array}\right),
$$

with $j_{i j}=j_{j i}^{*}$. Correspondingly, the persistent current is given by

$$
I=2 \operatorname{Re}\left(\rho_{21} j_{12}+\rho_{20} j_{02}\right)=2 \operatorname{Re}\left(\rho_{21} j_{12}+e^{-i \omega t} \widetilde{\rho}_{20} j_{02}\right) .
$$

The first term in the right-hand side of Eq. (36) is timeindependent and will be referred to as the dc component of the current, the second term in the right hand side of Eq. (36) is the ac component of the current. In a perfect ring $j_{20}=0$, thus an ac component in the current is a signature of ring distortion.

Figures 4 and 5 show the dc component $2 \operatorname{Re}\left(\rho_{21} j_{12}\right)$ and the amplitude of the ac component $2\left|\operatorname{Re}\left(\widetilde{\rho}_{20} j_{02}\right)\right|$ of the radiation-induced current for two values of the radiation intensity and different distortion radii. The exact steady state solutions of Eqs. (24)-(32) for these plots were found numerically. In the case of a high excitation power (Fig. 4) broad current peaks are observed. In the almost perfect ring $\left[r_{2} / r_{1}=0.99\right.$ curve in Fig. 4(a) $]$ the dc component has a single resonance peak. As the distortion degree increases, this peak shifts to a higher energy and its amplitude decreases (see $r_{2} / r_{1}=0.75$ curve). For a stronger distortion $\left(r_{2} / r_{1}=0.5\right)$ a second peak appears at a lower energy, which
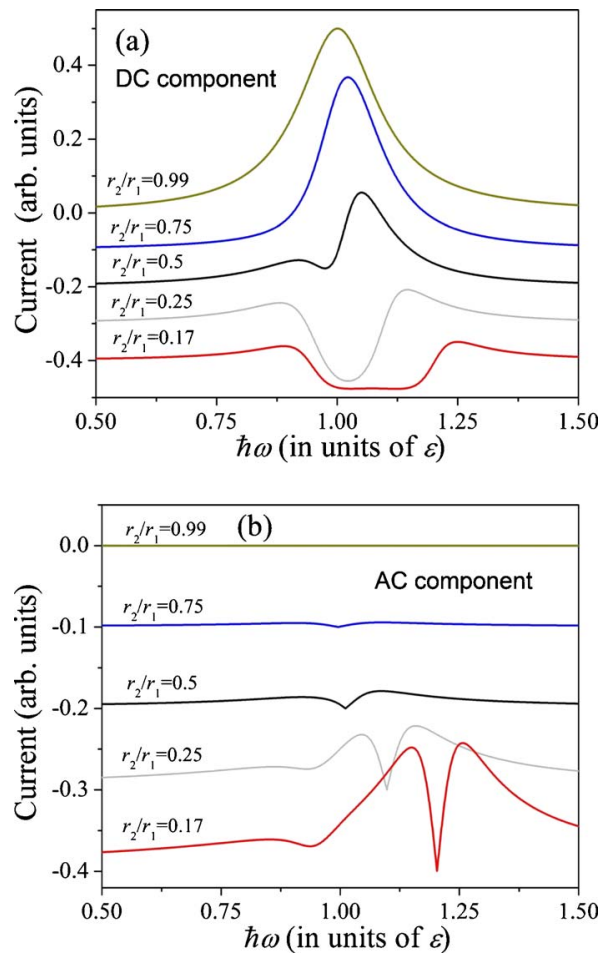

FIG. 4. (Color online) (a) dc component and (b) the amplitude of the ac component of the radiation-induced current vs photon energy for different distorted rings with the distortion parameter $\varphi$ $=\pi / 4$ at high radiation power. Curves other than $r_{2} / r_{1}=0.99$ were displaced for clarity. These plots were obtained using the parameters values $\kappa_{i, j}=0.001 \hbar / \varepsilon, \gamma_{i, j}=0.001 \hbar / \varepsilon, e E_{0} r_{1} / \varepsilon=0.1$.

is related to the lower energy split level $E_{1}$. At $r_{2} / r_{1}=0.25$ and $r_{2} / r_{1}=0.17$ an additional negative current peak is observed. The amplitude of the ac component of the persistent current is zero in the perfect ring. This amplitude becomes different than zero in distorted quantum rings with a maximum located in the region of the $E_{2}-E_{0}$ resonance (Fig. 4). The complex dependence of the dc and ac persistent current components on the radiation frequency indicates that significant quantum-interference effects are occurring.

The current peaks are narrower in the case of low radiation power (see Fig. 5). Figure 5(a) shows that the dc component is suppressed in quantum rings with strong distortion. In contrast, the amplitude of the ac component becomes nonzero in the distorted rings and increases with the distortion [Fig. 5(b)]. The maximum of the ac component is located in the region of the $E_{2}-E_{0}$ resonance, as in the case of the high radiation power. We note that the sign change of $\operatorname{Re}\left(\widetilde{\rho}_{20} j_{02}\right)$ is responsible for the vertical lines in the peak centers in Fig. $5(\mathrm{~b})$.

In the regime of low radiation power we can find an approximate solution of Eqs. (24)-(32). Eq. (31) gives the following expression for $\tilde{\rho}_{20}$ in the first order in $E_{0}$ :

$$
\tilde{\rho}_{20}=-\frac{i \hbar}{E_{2}-E_{0}-\hbar \omega-i \hbar \gamma_{20}} v_{20+} \rho_{00} .
$$

Similarly, from Eq. (27) $\tilde{\rho}_{01}$ in the first order in $E_{0}$ can be found. This expression for $\widetilde{\rho}_{01}$ together with Eq. (37) and Eq. (32) yields in the second order in $E_{0}$ 

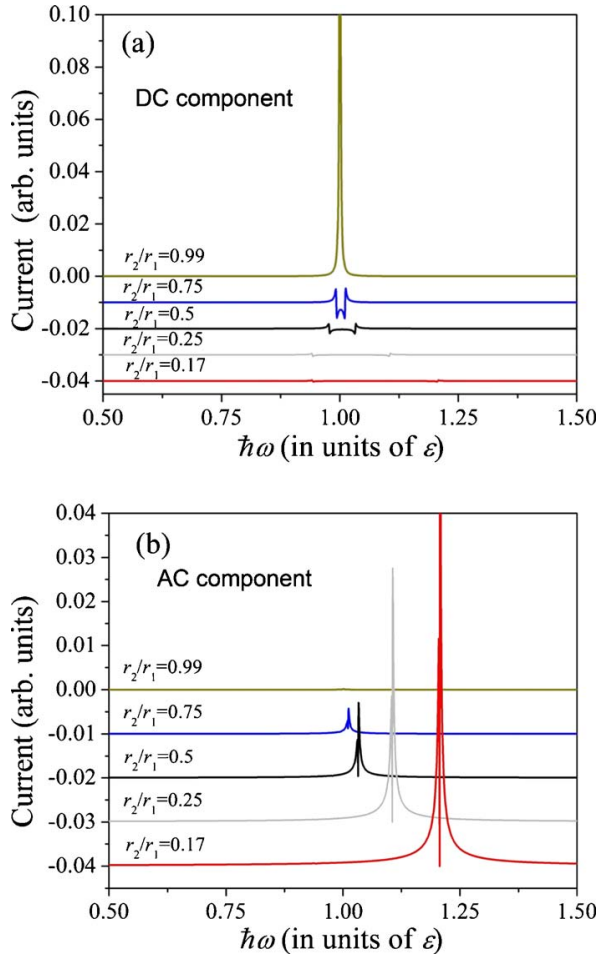

FIG. 5. (Color online) (a) dc component and (b) the amplitude of the ac component of the radiation-induced current vs photon energy at low radiation power. All calculation parameters are the same with those in Fig. 4 except $e E_{0} r_{1} / \varepsilon=0.001$.

$$
\begin{aligned}
\rho_{21}= & \frac{i \hbar}{E_{2}-E_{1}-i \hbar \gamma_{21}}\left[\frac{i \hbar}{E_{2}-E_{0}-\hbar \omega-i \hbar \gamma_{20}}\right. \\
& \left.+\frac{i \hbar}{E_{1}-E_{0}-\hbar \omega-i \hbar \gamma_{01}}\right] v_{01-v_{20+} \rho_{00} .}
\end{aligned}
$$

We have found that the persistent current components calculated from Eqs. (36)-(38) with $\rho_{0,0}=1$ perfectly coincides with the persistent current components calculated numerically in Fig. 5. In the case of a perfect ring, the current can be seen as a $\mathcal{X}_{2}$ effect. The presence of a distorsion induces a $\mathcal{X}_{1}$ term which corresponds to the ac component.

\section{CONCLUSIONS}

In conclusion, we have investigated persistent and radiation-induced currents in quantum rings with distortions.
We have derived an effective Schrödinger equation describing electrons in a narrow distorted quantum ring (closed loop) in the presence of an external magnetic field flux. We have shown that the electron energy spectrum is a periodic function of the magnetic flux. The ring curvature enters into the effective equations through a geometrical potential term. We have solved the equations in the case of a distorted ring consisting of four constant-curvature segments. We have considered the effect of the ring distortion on the magnetic flux-induced and radiation-induced currents. It was found that the effect on the flux-induced persistent current is more pronounced in quantum rings with a small number of electrons and lower chemical potential. The gaps at the points of intersection of the energy levels lead to a smoothing of the persistent current oscillations and to a different temperature dependence. The persistent current in a distorted ring with a large number of electrons behaves as it does in a perfect ring with a different radius in a renormalized magnetic field. Our choice of the distortion shape leads to a geometrical potential that corresponds to a square well in the curvilinear coordinates. For more realistic distortion shapes we expect some quantitative deviations in the results. However, in the persisten current case, the square well can be considered as an effective potential which describes a more complicated geometrical potential. In fact, the length and depth of the well can be chosen in such a way to reproduce the energy of the shallow bound states of a real distortion. This considerably simplifies the calculation of the current.

We have also found that the ring distortion affects radiation-induced currents. Using a density matrix approach and the rotating wave approximation, it was found that the current in distorted quantum rings acquires an ac component, in addition to the dc component characteristic of perfect rings. The frequency dependence of the dc component is modified by the distortion and shows several peaks of different sign. The frequency of the ac component is equal to the radiation frequency while its amplitude increases with the distortion. Finally, we remark that the nontrivial dependence of the dc persistent current component on the radiation frequency can be useful for quantum control schemes involving localized spins, as suggested in Ref. 22.

\section{ACKNOWLEDGMENTS}

We thank Prof. M. Dykman for many fruitful discussions. This research was supported by the National Science Foundation, Grant No. NSF DMR-0312491.
${ }^{1}$ A. G. Aronov and Y. S. Sharvin, Rev. Mod. Phys. 59, 755 (1987);

I. V. Krive and A. S. Rozhavsky, Int. J. Mod. Phys. B 6, 1255 (1992).

${ }^{2}$ J. Splettstoesser, M. Governale, and U. Zülicke, Phys. Rev. B 68, 165341 (2003).

${ }^{3}$ I. D. Vagner, A. S. Rozhavsky, P. Wyder, and A. Yu. Zyuzin, Phys. Rev. Lett. 80, 2417 (1998).

${ }^{4}$ S. N. Shevchenko, Yu. V. Pershin, and I. D. Vagner, Physica E
(Amsterdam) 24, 82 (2004).

${ }^{5}$ T. V. Shahbazyan and S. E. Ulloa, Phys. Rev. B 55, 13702 (1997).

${ }^{6}$ T.-Z. Qian, Y.-S. Yi, and Z.-B. Su, Phys. Rev. B 55, 4065 (1997).

${ }^{7}$ M. Büttiker, Y. Imry, and R. Landauer, Phys. Lett. 96A, 365 (1983).

${ }^{8}$ H. F. Cheung, Y. Gefen, E. K. Riedel, and W. H. Shih, Phys. Rev. B 37, 6050 (1988). 
${ }^{9}$ Y. Meir, Y. Gefen, and O. Entin-Wohlman, Phys. Rev. Lett. 63, 798 (1989).

${ }^{10}$ O. Entin-Wohlman, Y. Gefen, Y. Meir, and Y. Oreg, Phys. Rev. B 45, 11890 (1992).

${ }^{11}$ H. Mathur and A. D. Stone, Phys. Rev. B 44, 10957 (1991).

${ }^{12}$ L. P. Levy, G. Dolan, J. Dunsmuir, and H. Bouchiat, Phys. Rev. Lett. 64, 2074 (1990).

${ }^{13}$ V. Chandrasekhar, R. A. Webb, M. J. Brady, M. B. Ketchen, W. J. Gallagher, and A. Kleinsasser, Phys. Rev. Lett. 67, 3578 (1991).

${ }^{14}$ D. Mailly, C. Chapelier, and A. Benoit, Phys. Rev. Lett. 70, 2020 (1993).

${ }^{15}$ P. Mohanty, Ann. Phys. 8, 549 (1999).

${ }^{16}$ E. M. Q. Jariwala, P. Mohanty, M. B. Ketchen, and R. A. Webb, Phys. Rev. Lett. 86, 1594 (2001).

${ }^{17}$ D. Loss and T. Martin, Phys. Rev. B 47, 4619 (1993).

${ }^{18}$ D. Wohlleben, M. Esser, P. Freche, E. Zipper, and M. Szopa, Phys. Rev. Lett. 66, 3191 (1991).

${ }^{19}$ L. I. Magarill, D. A. Romanov, and A. V. Chaplik, Zh. Eksp. Teor. Fiz. 110, 669 (1996) [JETP 83, 361 (1996)].
${ }^{20}$ D. V. Bulaev, V. A. Geyler, and V. A. Margulis, Phys. Rev. B 69, 195313 (2004).

${ }^{21}$ V. Gudmundsson, C.-S. Tang, and A. Manolescu, Phys. Rev. B 67, 161301(R) (2003).

${ }^{22}$ Yu. V. Pershin and C. Piermarocchi, cond-mat/0502001 (unpublished).

${ }^{23}$ A. Matos-Abiague and J. Berakdar, Phys. Rev. Lett. 94, 166801 (2005).

${ }^{24}$ R. C. T. da Costa, Phys. Rev. A 23, 1982 (1981).

${ }^{25} \mathrm{~S}$. N. Shevchenko and Yu. A. Kolesnichenko, JETP 92, 811 (2001).

${ }^{26}$ D. Loss, Phys. Rev. Lett. 69, 343 (1992).

${ }^{27}$ M. Moskalets, Physica E (Amsterdam) 4, 17 (1999); Eur. Phys. J. B 7, 645 (1999).

${ }^{28}$ Quantum Coherence, Correlation and Decoherence in Semiconductor Nanostructures, edited by T. Takagahara (Elsevier Science, Oxford, 2003), ch. 9. 